\title{
Prophylactic percutaneous circulatory support in high risk transcatheter aortic valve implantation
}

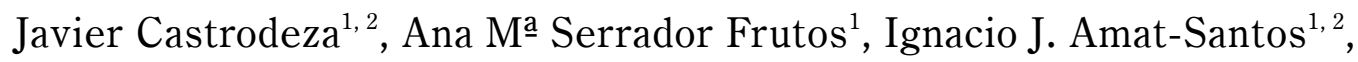 \\ Inés Sayago Silva ${ }^{1}$, José Alberto San Román ${ }^{1,2}$ \\ ${ }^{1}$ Department of Cardiology, Institute of Heart Sciences (ICICOR), \\ Hospital Clínico Universitario, Valladolid, Spain \\ ${ }^{2}$ CIBER de Enfermedades Cardiovasculares, Spain
}

Mechanical circulatory support in transcatheter aortic valve implantation (TAVI) procedures is a useful solution for both planned and in emergent cases and provides prophylactic implantations which can subsequently avoid complications. Venoarterial extracorporeal membrane oxygenation (VA-ECMO) provides both hemodynamic and oxygenation support. Percutaneous VA-ECMO can be inserted with fluoroscopy or ultrasound guidance. A perfusion cannula is required to protect against distal limb ischemia. VA-ECMO can either be used in prophylactic settings or as a rescue therapy [1-4].

Reported herein, is a prophylactic VA-ECMO paradigm case in a TAVI setting in order to illustrate the main steps. Also, a systematic search of all existing literature was performed from the main bibliographic databases (Pubmed, Medline, Google Scholar, and Cochrane).

The case of a 63-year-old male patient with ischemic cardiomyopathy, severe left ventricular systolic dysfunction, dual-chamber implantable cardiac defibrillator and moderate aortic stenosis is presented. Two months prior to index admission, he developed chest pain and dyspnea at minimal exertion. A coronary angiogram revealed significant stenosis of the distal left main coronary artery including the origin of the descendent coronary artery and the circumflex and the echocardiogram showed a severe aortic stenosis (peak velocity of $3.2 \mathrm{~m} / \mathrm{s}$, peak and mean gradients of 40 and $23 \mathrm{mmHg}$ respectively, estimated area of $0.9 \mathrm{~cm}^{2}$ ), functional moderate mitral regurgitation with effective regurgitant orifice area of $0.3 \mathrm{~cm}^{2}$, and left ventricular ejection fraction (LVEF) of $22 \%$. A cardiac computed tomography (CT) showed an Agatston score of the aortic valve of 3568. Lowflow low-gradient severe aortic stenosis diagnosis was made in the presence of low LVEF on the basis of the calcium score. Dobutamine stress echocardiogram was not contemplated due to coronary disease. Aortic-ileo-femoral axis CT revealed both femoral accesses above $6 \mathrm{~mm}$. The Heart Team decided on percutaneous intervention (TAVI + coronary intervention) under VA-ECMO support due to the high surgical risk (LogEuroScore 28\%). A staged procedure was planned for percutaneous VA-ECMO implantation (CardioHelp System ${ }^{\circledR}$, Maquet, Germany) via left femoral access (15 F arterial cannula, $23 \mathrm{~F}$ venous cannula) with perfusion cannula $(6 \mathrm{~F})$ connected between the arterial cannula and a distal sheath in the femoral artery. It was programmed at $3000 \mathrm{rpm}$, with an estimated flow of $2.6-2.8 \mathrm{~L} / \mathrm{min}$. A bolus of $10,000 \mathrm{U}$ of unfractioned heparin was given. Invasive hemodynamics were monitored throughout the procedure. The bifurcation between the left main-circumflex and left main-anterior descendent coronary artery was treated with two drug-eluting stents, finishing with a kissing-balloon technique. A $34 \mathrm{~mm}$ CoreValve Evolut-R (Corevalve Evolute ${ }^{\circledR}$, Medtronic Inc., $\mathrm{MN}$, USA) was deployed via right femoral artery, requiring post-dilatation with a $25 \mathrm{~mm}$ True Dilatation Balloon (Bard Peripheral Vascular, Inc., USA). The cannulas were clamped sequentially and removed. Femoral sites were both closed with the

Address for correspondence: Javier Castrodeza, MD, Institute of Heart Sciences, Hospital Clínico Universitario, Ramón y Cajal 3. 47005, Valladolid, Spain, tel: 00349834200 26, fax: 00349832553 05, e-mail: jcastrodeza5@gmail.com 
Perclose ProGlide ${ }^{\circledast}$ suture system. There was an improvement in pre-discharge LVEF (around 34\%). Femoral ultrasound confirmed no vascular complications. The patient was discharged uneventfully and remains asymptomatic at 6 month follow-up.

Little has been said regarding the prophylactic use of VA-ECMO before a combined complex TAVI and coronary procedure. Table 1 summarizes the main studies. A series with 14 VA-ECMO cases showed a survival rate of $50 \%$ in 4 cases with severe aortic valve disease [1]. A later series included a comparison between emergency versus planned ECMO in high risk patients, suggesting that outcomes remain better if ECMO is implanted beforehand [2]. In this report, severely impaired LVEF, slow recovery from rapid left ventricular pacing, high vasopressor requirements or a concomitant high-risk percutaneous coronary intervention were suggested to be suitable scenarios for VA-ECMO prophylactic implantation. The rate of procedural success with planned ECMO in this study reached $100 \%$ with no evidence of 30-day mortality. In spite of such promising results, times of fluoroscopy were longer and up to $11 \%$ had vascular complications. Vascular complications can be inevitably seen as a concern in procedures requiring bilateral femoral access with large sheaths but can be minimized by a careful selection of potential candidates, low diameter cannulas and percutaneous guided approach [3]. Eligible patients usually remain stable with a flow of $2 \mathrm{~L} / \mathrm{min}$ and this complementary support can be achieved with low profile cannulas. Additionally, third generation TAVI devices include introducers of $14 \mathrm{~F}$ which lead to reduce vascular complications.

In a series published by Seco et al. [4], some pre-procedural aspects might tip the balance for prophylactic ECMO, such as unstable heart failure pre-TAVI requiring support, hemodynamic instability after balloon aortic valvuloplasty, or poor baseline hemodynamic parameters. Better results might be obtained with prophylactic versus rescue implantation, despite a high risk of acute kidney injury $(\sim 36 \%)$ in the rescue group. For all of the above, the main conclusion was that results with a planned ECMO in a high-risk TAVI procedure is comparable to standard TAVI procedures [2, 4]. In a planned scenario, someone could argue that a cardiopulmonary bypass machine is cheaper, with less risk of bleeding complications, and avoids the increased afterload made by VA-ECMO by placing a Vent cannula. Unfortunately, it is not available in every hospital and cannot avoid a later VAECMO implantation if coming off it is not achieved.

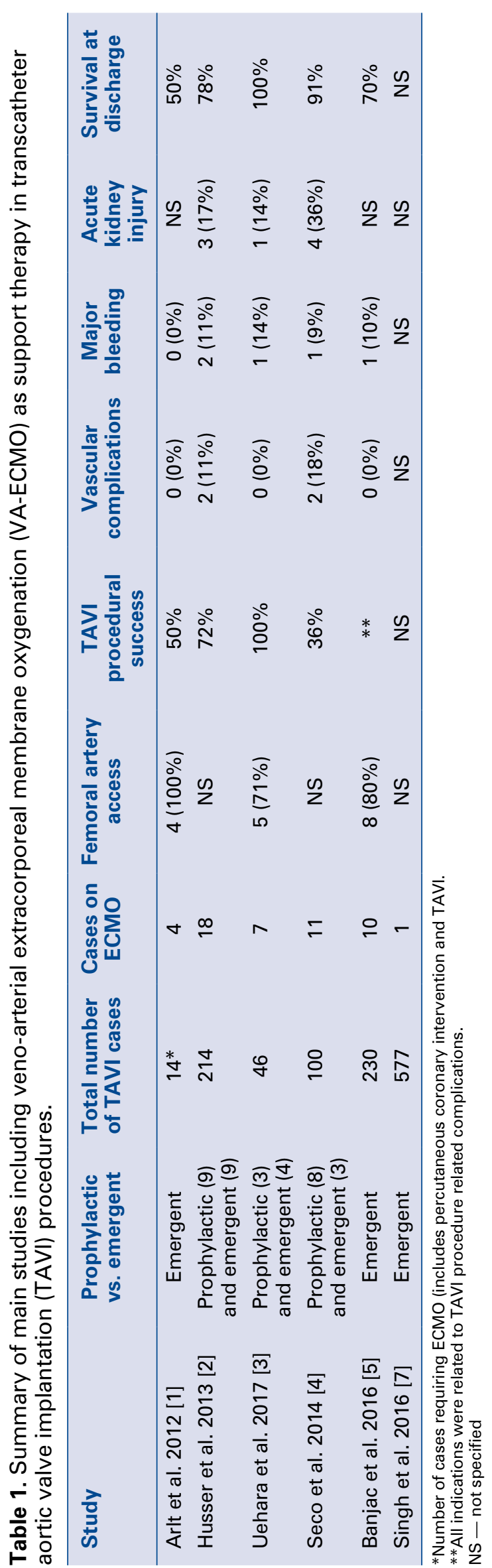


Regarding VA-ECMO as a rescue therapy, Banjac et al. [5] report an incidence of $4.3 \%$ of TAVI procedures due to hemodynamic collapse, with an in-hospital mortality up to $30 \%$. Even in emerging valve-in-valve procedures it is a feasible option for gaining time and deployment of the valve [6,7]. A recent registry showed an incidence of mechanical circulatory support use of $0.6 \%$ in TAVI procedures with differences between elective $(0.5 \%)$ and emergent cases (1.2\%) [8]. Hemolysis and thrombocytopenia are recognizable complications during ECMO, with an increasing incidence in relation to time on support [9].

Percutaneous VA-ECMO can be seen as an alternative to other devices and is of great help in aortic stenosis scenarios [10], especially in high volume centres with experienced operators.

In the current transition from surgical to percutaneous treatment of aortic stenosis in more complex contexts, not only acceptable results in intermediate and low risk patients are requested but also in high risk once as well. There are limited data and few case studies supporting the use of percutaneous VA-ECMO in these scenarios, assuming a higher risk of vascular complications and obviously, extra costs thereby derived.

\section{Conflict of interest: None declared}

\section{References}

1. Arlt M, Philipp A, Voelkel S, et al. Early experiences with miniaturized extracorporeal life-support in the catheterization laboratory. Eur J Cardiothorac Surg. 2012; 42(5): 858-863, doi: 10.1093/ /ejcts/ezs176, indexed in Pubmed: 22555310.

2. Husser O, Holzamer A, Philipp A, et al. Emergency and prophylactic use of miniaturized veno-arterial extracorporeal membrane oxygenation in transcatheter aortic valve implantation. Catheter Cardiovasc Interv. 2013; 82(4): E542-E551, doi: 10.1002/ /ccd.24806, indexed in Pubmed: 23554044.

3. Uehara K, Minakata K, Saito N, et al. Use of extracorporeal membrane oxygenation in complicated transcatheter aortic valve replacement. Gen Thorac Cardiovasc Surg. 2017; 65(6): 329 -336, doi: 10.1007/s11748-017-0757-1, indexed in Pubmed: 28236098.

4. Seco M, Forrest P, Jackson SA, et al. Extracorporeal membrane oxygenation for very high-risk transcatheter aortic valve implantation. Heart Lung Circ. 2014; 23(10): 957-962, doi: 10.1016/j. hlc.2014.05.006, indexed in Pubmed: 24954708.

5. Banjac I, Petrovic M, Akay MH, et al. Extracorporeal membrane oxygenation as a procedural rescue strategy for transcatheter aortic valve replacement cardiac complications. ASAIO J. 2016; 62(1): e1-e4, doi: 10.1097/MAT.0000000000000275, indexed in Pubmed: 26309098.

6. Summers MR, Mick S, Kapadia SR, et al. Emergency valve-invalve transcatheter aortic valve replacement in a patient with degenerated bioprosthetic aortic stenosis and cardiogenic shock on veno-arterial extracorporeal membrane oxygenation. Catheter Cardiovasc Interv. 2018; 92(3): 592-596, doi: 10.1002/ccd.26990, indexed in Pubmed: 28296034.

7. Singh V, Damluji AA, Mendirichaga R, et al. Elective or emergency use of mechanical circulatory support devices during transcatheter aortic valve replacement. J Interv Cardiol. 2016; 29(5): 513-522, doi: 10.1111/joic.12323, indexed in Pubmed: 27550213.

8. Kolte D, Khera S, Vemulapalli S, et al. Outcomes Following Urgent/Emergent Transcatheter Aortic Valve Replacement: Insights From the STS/ACC TVT Registry. JACC Cardiovasc Interv. 2018; 11(12): 1175-1185, doi: 10.1016/j.jcin.2018.03.002, indexed in Pubmed: 29929641.

9. Murphy DA, Hockings LE, Andrews RK, et al. Extracorporeal membrane oxygenation-hemostatic complications. Transfus Med Rev. 2015; 29(2): 90-101, doi: 10.1016/j.tmrv.2014.12.001, indexed in Pubmed: 25595476.

10. Makdisi G, Makdisi PB, Wang IW. New horizons of non-emergent use of extracorporeal membranous oxygenator support. Ann Transl Med. 2016; 4(4): 76, doi: 10.3978/j.issn.23055839.2016.02.04, indexed in Pubmed: 27004223. 